\title{
Eosin Y Catalyzed Visible-Light-Promoted Aerobic Oxidative Cyclization of 2-Aminobenzothiazole
}

\author{
Vishal Srivastava, ${ }^{1}$ Pravin K. Singh, ${ }^{2}$ Praveen P. Singh ${ }^{3, *}$
}

\footnotetext{
1 Laboratory of Green Synthesis, Department of Chemistry, University of Allahabad, 211002 Allahabad, India

2 Food Analysis and Research Lab, Centre of Food Technology, University of Allahabad, 211002 Allahabad, India

3 Department of Chemistry, United College of Engineering \& Research, Naini, 211010 Allahabad, India

* Corresponding author's e-mail address: ppsingh23@gmail.com
}

RECEIVED: March 23, 2015 * ACCEPTED: May 4, 2015

Abstract: A mild and efficient one-pot visible light irradiated synthesis of 2-aminobenzothiazole 4(a-1) from arylisothiocyanate 1(a-1) and secondary amines 2 have been reported in presence of eosin $Y$ as an organophotoredox catalyst at room temperature under aerobic condition. This synthesis includes application of air and visible light as inexpensive, readily available, high atom economy, non-toxic and sustainable regents.

Keywords: Eosin Y, visible-light, organophotoredox, green chemistry, aerobic condition, oxidative cyclization, aminobenzothiazole, single electron transfer (SET).

\section{INTRODUCTION}

$\mathbf{T}$ HE potential of developing new synthetic methodologies using visible light has recently received much attention from a number of research groups. ${ }^{[1]}$ This is because solar energy (visible light) is clean, easy to handle and an unlimited energy source having great prospects for the development of sustainable and eco-friendly protocols for organic synthesis. ${ }^{[2]}$ The development of methods to efficiently harness the solar radiation energy has emerged as one of the central scientific challenges of the twenty first century. ${ }^{[3-5]}$ Therefore, some pioneering researchers have dedicated to converting solar energy into chemical energy for chemical transformations ${ }^{[6,7]}$ which includes a promising strategy for the application of photoredox catalysts to initiate single electron transfer processes have been developed. ${ }^{[8,9]}$ A surge of interest from the synthetic community has brought photoredox manifolds to the forefront of catalysis. In this sequence visible light photoredox catalysis has recently received much attention in organic synthesis owing to readily availability, sustainability, non-toxicity and ease of handling of visible light. ${ }^{[10-15]}$ In their revolutionary work in this area, MacMillan, ${ }^{[16]}$ Yoon ${ }^{[17]}$ and Stephenson ${ }^{[18-19]}$ have used Ruthenium and Iridium complexes as the photoredox catalyst, which has inspired the development of several powerful methods for various chemical transformations useful in organic synthesis.

However, these transition metal based photocatalysts disadvantageously exhibit high cost, low sustainability and potential toxicity. Recently, a superior alternative to transition metal photoredox catalysts, especially metal-free organic dyes such as eosin $Y$, fluorescein, Rose Bengal, Nile Red, Perylene and Rhodamine $B$ have been used as economically and ecologically superior surrogates for $\mathrm{Ru}(\mathrm{II})$ and Ir(II) complexes in visible-light promoted organic transformations involving SET ${ }^{[2-23]}$ (single electron transfer). These organic dyes have got much more attention with the last few years also due to easy handling, eco-friendly and have great potential for applications in visible-light-mediated organic synthesis ${ }^{[24-27]}$ which fulfils the basic principle of green chemistry.

Heterocycles bearing thiazole, sulphur and nitrogen moieties constitute the core structure of a number of pharmacologically and biologically active interesting compounds. Benzothiazole derivatives possess a wide spectrum of biological applications such as antitumor, 


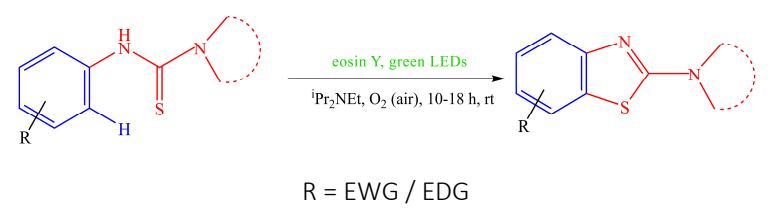

Scheme 1. Eosin $Y$ catalysed visible light promoted synthesis of 2-aminobenzothiazole.

schictosomicidal, anti-inflammatory, anticonvulsant, antidiabetic, antipsychotic, diuretic, and antimicrobial activities. $^{[28]}$

The synthesis of 2-aminobenzothiazole have not been reported by photooxidation reaction so far. Meanwhile the aerobic oxygen has received a great importance in research during present time. ${ }^{[29-30]}$ In general, organosulfur / nitrogen compounds have been frequently used as precursors in radical reactions because they form radicals very readily. ${ }^{[31-33]}$ Encouraged by organocatalytic visiblelight-mediated aerobic oxidative transformations ${ }^{[34,35]}$ and in continuation of our work on development of novel environmentally benign synthesis ${ }^{[3-39]}$ herein we report a simple, visible light irradiated, efficient and green protocol for the synthesis of 2-aminobenzothiazole, using eosin $Y$ as photocatalyst with excellent yield as depicted in Scheme 1.

\section{EXPERIMENTAL}

Melting points were determined by open glass capillary method and are uncorrected. All chemicals used were reagent grade and were used as received. ${ }^{1} \mathrm{H}$ NMR and ${ }^{13} \mathrm{C}$ NMR spectra were recorded on a Bruker AVANCE DPX (400 MHz and $75 \mathrm{MHz}$ ) FT spectrometer in DMSO using TMS as an internal reference (chemical shift in $\delta, \mathrm{ppm}$ ). Mass spectra were recorded on JEOL SX-303 (FAB) mass spectrophotometer. Elemental analyses were carried out using a Coleman automatic $\mathrm{C}, \mathrm{H}, \mathrm{N}$ analyser.

\section{General Procedure for the Synthesis of 2-Aminobenzothiazoles 4(a-l)}

A solution of an arylisothiocyanate $\mathbf{1}(\mathrm{a}-\mathrm{I})(1.0 \mathrm{mmol})$ and an secondary amine $2(1.0 \mathrm{mmol})$ in DMF $(3 \mathrm{~mL})$ was heated at $65{ }^{\circ} \mathrm{C}$ for $2-5 \mathrm{~h}$ to form the corresponding $\mathrm{N}$-arylthioureas (as monitored by TLC). Then, eosin Y ( $2.0 \mathrm{~mol} \%$ ) and $\mathrm{Pr}_{2} \mathrm{NEt}$ ( 2.0 equiv.) were added and the mixture was irradiated with green LEDs $(2.4 \mathrm{~W}, 120 \mathrm{~lm})$ with stirring under an air atmosphere at $\mathrm{rt}$ for $10-18 \mathrm{~h}$. After completion of the reaction (monitored by TLC), water $(5 \mathrm{~mL}$ ) was added and the mixture was extracted with EtOAc $(3 \times 5 \mathrm{~mL})$. The combined organic phase was dried over $\mathrm{MgSO}_{4}$, filtered and evaporated under reduced pressure. The resulting product was purified by silica gel column chromatography using a gradient mixture of hexane / ethyl acetate as eluent to afford an analytically pure $4(a-I)$. All the products are known compounds and were characterized by the comparison of their spectral data with those reported in the literature.

\section{4a. 4-(6-Chlorobenzo[d]thiazol-2-yl)morpholine} m.p. $160^{\circ} \mathrm{C}, \mathrm{m} / \mathrm{z}$ : 254.03; Mol. wt: 254.74; ${ }^{1} \mathrm{H}$ NMR (400 MHz, DMSO- $\left.d_{6}\right): \delta=3.57\left(\mathrm{~d}, 4 \mathrm{H}, \mathrm{N}-\mathrm{CH}_{2}-\right), 3.65\left(\mathrm{~d}, 4 \mathrm{H}, \mathrm{O}-\mathrm{CH}_{2}-\right.$ ), 7.56-8.13 (d, 3H, ArH), ${ }^{13} \mathrm{CNMR}\left(75 \mathrm{MHz}\right.$, DMSO- $\left.d_{6}\right): \delta=48.4$, 66.3, 118.3, 121.2, 125.8, 129.8, 132.3, 151.3, 168.0; Anal. calcd for $\mathrm{C}_{11} \mathrm{H}_{11} \mathrm{CIN}_{2} \mathrm{OS}$ : C, 51.86: $\mathrm{H}, 4.35: \mathrm{N}, 11.00$. Found: C, 51.84: H, 4.32; N, 10.98 .

\section{4b. 4-(6-Nitrobenzo[d]thiazol-2-yl)morpholine} m.p. $155^{\circ} \mathrm{C}, \mathrm{m} / \mathrm{z}$ : 265.05; Mol. wt: 265.29; ${ }^{1} \mathrm{H}$ NMR (400 MHz, DMSO- $\left.d_{6}\right): \delta=3.57\left(\mathrm{~d}, 4 \mathrm{H}, \mathrm{N}-\mathrm{CH}_{2}-\right), 3.65\left(\mathrm{~d}, 4 \mathrm{H}, \mathrm{O}-\mathrm{CH}_{2}-\right.$ ), 8.01-8.62 (d, $3 \mathrm{H}, \mathrm{ArH}),{ }^{13} \mathrm{C}$ NMR $\left(75 \mathrm{MHz}\right.$, DMSO- $\left.d_{6}\right): \delta=48.4$, 66.3, 117.3, 119.1, 121.3, 131.3, 144.3, 159.3, 168.0; Anal. calcd for $\mathrm{C}_{11} \mathrm{H}_{11} \mathrm{~N}_{3} \mathrm{O}_{3} \mathrm{~S}: \mathrm{C}, 49.80: \mathrm{H}, 4.18: \mathrm{N}, 15.84$. Found: $\mathrm{C}$, 49.78: H, 4.16; N, 15.82.

4c. 4-(6-Methylbenzo[d]thiazol-2-yl)morpholine m.p. $140{ }^{\circ} \mathrm{C}, \mathrm{m} / \mathrm{z}$ : 234.08; Mol. wt: $234.32 ;{ }^{1} \mathrm{H}$ NMR (400 MHz, DMSO- $\left.d_{6}\right): \delta=2.34\left(\mathrm{~s}, 3 \mathrm{H},-\mathrm{CH}_{3}-\right), 3.57\left(\mathrm{~d}, 4 \mathrm{H}, \mathrm{N}-\mathrm{CH}_{2}-\right), 3.65$ (d, 4H, O- $\mathrm{CH}_{2}-$ ) , 7.33-7.89 (d, 3H, ArH), ${ }^{13} \mathrm{C}$ NMR (75 MHz, DMSO- $\left.d_{6}\right): \delta=20.9,48.4,66.3,117.1,121.3,126.6,130.7$, 134.1, 150.2, 168.0; Anal. calcd for $\mathrm{C}_{12} \mathrm{H}_{14} \mathrm{~N}_{2}$ OS: C, 61.51: $\mathrm{H}$, 6.02: N, 11.96. Found: C, 61.49: H, 6.00; N, 11.94.

\section{4d. 4-(6-Methoxybenzo[d]thiazol-2-yl)morpholine} m.p. $135^{\circ} \mathrm{C}, \mathrm{m} / \mathrm{z}$ : 250.08; Mol. wt: 250.32; ${ }^{1 \mathrm{H}} \mathrm{NMR}(400 \mathrm{MHz}$, DMSO- $\left.d_{6}\right): \delta=3.57$ (d, 4H, N-CH - ) $3.65\left(\mathrm{~d}, 4 \mathrm{H}, \mathrm{O}-\mathrm{CH}_{2}-\right.$ ), $3.83\left(\mathrm{~s}, 3 \mathrm{H},-\mathrm{OCH}_{3}-\right), 7.00-7.53(\mathrm{~d}, 3 \mathrm{H}, \mathrm{ArH}),{ }^{13} \mathrm{C} \mathrm{NMR}$ (75 MHz, DMSO- $\left.d_{6}\right): \delta=48.4,55.8,66.3,104.9,114.6$, 118.2, 131.9, 145.5, 156.7, 168.0; Anal. calcd for $\mathrm{C}_{12} \mathrm{H}_{14} \mathrm{~N}_{2} \mathrm{O}_{2} \mathrm{~S}: \mathrm{C}, 57.58: \mathrm{H}, 5.64: \mathrm{N}, 11.19$. Found: $\mathrm{C}, 57.56: \mathrm{H}$, 5.62; N, 11.17 .

\section{4e. 6-Chloro-2-(piperidin-1-yl)benzo[d]thiazole} m.p. $170{ }^{\circ} \mathrm{C}, \mathrm{m} / \mathrm{z}$ : 252.05; Mol. wt: 252.76; ${ }^{1} \mathrm{H}$ NMR $(400 \mathrm{MHz}$, DMSO- $\left.d_{6}\right): \delta=1.53\left(\mathrm{~d}, 4 \mathrm{H}\right.$, aliphatic $\left.-\mathrm{CH}_{2}-\right), 1.59(\mathrm{~d}, 2 \mathrm{H}$, aliphatic $\left.-\mathrm{CH}_{2}-\right), 3.71\left(\mathrm{~d}, 4 \mathrm{H}, \mathrm{N}-\mathrm{CH}_{2}-\right), 7.56-8.13(\mathrm{~d}, 3 \mathrm{H}, \mathrm{ArH}),{ }^{13} \mathrm{C}$ NMR $\left(75 \mathrm{MHz}\right.$, DMSO- $\left.d_{6}\right): \delta=24.5,25.5,54.5,118.3,121.2$, 125.8, 129.8, 132.3, 151.3, 168.0; Anal. calcd for $\mathrm{C}_{12} \mathrm{H}_{13} \mathrm{ClN}_{2} \mathrm{~S}$ : C, 57.02: H, 5.18: N, 11.08. Found: C, 57.00: H, 5.16; N, 11.05 .

\section{4f. 6-Nitro-2-(piperidin-1-yl)benzo[d]thiazole} m.p. $166^{\circ} \mathrm{C}, \mathrm{m} / \mathrm{z}$ : 263.07; Mol. wt: 263.32; ${ }^{1 \mathrm{H}} \mathrm{NMR}(400 \mathrm{MHz}$, DMSO- $\left.d_{6}\right): \delta=1.53\left(\mathrm{~d}, 4 \mathrm{H}\right.$, aliphatic $\left.-\mathrm{CH}_{2}-\right), 1.59(\mathrm{~d}, 2 \mathrm{H}$, aliphatic $-\mathrm{CH}_{2}-$ ), 3.71 (d, $\left.4 \mathrm{H}, \mathrm{N}-\mathrm{CH}_{2}-\right), 8.01-8.62(\mathrm{~d}, 3 \mathrm{H}, \mathrm{ArH})$, ${ }^{13} \mathrm{C} \mathrm{NMR}\left(75 \mathrm{MHz}\right.$, DMSO- $\left.d_{6}\right): \delta=24.5,25.5,54.5,117.3,119.1$, 121.3, 131.3, 144.3, 159.3, 168.0; Anal. calcd for $\mathrm{C}_{12} \mathrm{H}_{13} \mathrm{~N}_{3} \mathrm{O}_{2} \mathrm{~S}$ : C, 54.74: H, 4.98: N, 15.96. Found: C, 54.72: H, 4.96; N, 15.94 . 
4g. 6-Methyl-2-(piperidin-1-yl)benzo[d]thiazole m.p. $155{ }^{\circ} \mathrm{C}, \mathrm{m} / \mathrm{z}$ : 232.10; Mol. wt: 232.34; ${ }^{1} \mathrm{H}$ NMR $\left(400 \mathrm{MHz}, \mathrm{DMSO}-d_{6}\right): \delta=1.53\left(\mathrm{~d}, 4 \mathrm{H}\right.$, aliphatic $\left.-\mathrm{CH}_{2}-\right), 1.59$ (d, $2 \mathrm{H}$, aliphatic $\left.-\mathrm{CH}_{2}-\right), 2.34\left(\mathrm{~s}, 3 \mathrm{H},-\mathrm{CH}_{3}\right), 3.71(\mathrm{~d}, 4 \mathrm{H}$, $\mathrm{N}-\mathrm{CH}_{2}-$ ), 7.33-7.89(d, 3H, ArH), ${ }^{13} \mathrm{C}$ NMR (75 MHz, DMSO- $\left.d_{6}\right): \delta=20.9,24.5,25.5,54.5,117.1,121.3,126.6$, 130.7, 134.1, 150.2, 168.0; Anal. calcd for $\mathrm{C}_{13} \mathrm{H}_{16} \mathrm{~N}_{2} \mathrm{~S}$ : C, 67.20: H, 6.94: N, 12.06. Found: C, 67.18: H, 6.93; N, 12.04 .

\section{4h. 6-Methoxy-2-(piperidin-1-yl)benzo[d]thiazole} m.p. $140{ }^{\circ} \mathrm{C}, \mathrm{m} / \mathrm{z}$ : 248.10; Mol. wt: 248.34; ${ }^{1 \mathrm{H}} \mathrm{NMR}$ $\left(400 \mathrm{MHz}, \mathrm{DMSO}-d_{6}\right): \delta=1.53\left(\mathrm{~d}, 4 \mathrm{H}\right.$, aliphatic $\left.-\mathrm{CH}_{2}-\right), 1.59$ (d, $2 \mathrm{H}$, aliphatic $\left.-\mathrm{CH}_{2}-\right), 3.71\left(\mathrm{~d}, 4 \mathrm{H}, \mathrm{N}-\mathrm{CH}_{2}-\right), 3.83(\mathrm{~s}, 3 \mathrm{H}$, $\left.-\mathrm{CH}_{3}\right), 7.00-7.53$ (d, 3H, ArH), ${ }^{13} \mathrm{C}$ NMR (75 MHz, DMSO- $d_{6}$ ): $\delta=24.5,25.5,54.5,55.8,104.9,114.6,118.2,131.9,145.5$, 156.7, 168.0; Anal. calcd for $\mathrm{C}_{13} \mathrm{H}_{16} \mathrm{~N}_{2} \mathrm{OS}$ : C, 62.87: $\mathrm{H}, 6.49$ : $\mathrm{N}, 11.28$. Found: $\mathrm{C}, 62.85: \mathrm{H}, 6.47 ; \mathrm{N}, 11.26$.

\section{4i. 6-Chloro-2-(pyrrolidin-1-yl)benzo[d]thiazole} m.p. $167{ }^{\circ} \mathrm{C}, \mathrm{m} / \mathrm{z}$ : 238.03; Mol. wt: 238.74; ${ }^{1 \mathrm{H}} \mathrm{NMR}$ $\left(400 \mathrm{MHz}, \mathrm{DMSO}-d_{6}\right): \delta=1.92\left(\mathrm{~d}, 4 \mathrm{H}\right.$, aliphatic $\left.-\mathrm{CH}_{2}-\right), 3.44$ (d, $4 \mathrm{H}, \mathrm{N}-\mathrm{CH}_{2}-$ ) , 7.56-8.13 (d, 3H, ArH), ${ }^{13} \mathrm{C} \mathrm{NMR}(75 \mathrm{MHz}$, DMSO- $\left.d_{6}\right): \delta=25.5,54.1,118.3,121.2,125.8,129.8,132.3$, 151.3, 168.0; Anal. calcd for $\mathrm{C}_{11} \mathrm{H}_{11} \mathrm{CIN}_{2} \mathrm{~S}$ : C, 55.34: H, 4.64: $\mathrm{N}, 11.73$. Found: $\mathrm{C}, 55.32: \mathrm{H}, 4.62 ; \mathrm{N}, 11.70$.

\section{4j. 6-Nitro-2-(pyrrolidin-1-yl)benzo[d]thiazole} m.p. $145{ }^{\circ} \mathrm{C}, \mathrm{m} / \mathrm{z}$ : 249.06; Mol. wt: 249.29; ${ }^{1} \mathrm{H}$ NMR $\left(400 \mathrm{MHz}, \mathrm{DMSO}-d_{6}\right): \delta=1.92\left(\mathrm{~d}, 4 \mathrm{H}\right.$, aliphatic $\left.-\mathrm{CH}_{2}-\right)$, $3.44\left(\mathrm{~d}, 4 \mathrm{H}, \mathrm{N}-\mathrm{CH}_{2}-\right), 8.01-8.62(\mathrm{~d}, 3 \mathrm{H}, \mathrm{ArH}),{ }^{13} \mathrm{C} \mathrm{NMR}$ $\left(75 \mathrm{MHz}, \mathrm{DMSO}-d_{6}\right): \delta=25.5,54.1,117.3,119.1,121.3$, 131.3, 144.3, 159.3, 168.0; Anal. calcd for $\mathrm{C}_{11} \mathrm{H}_{11} \mathrm{~N}_{3} \mathrm{O}_{2} \mathrm{~S}$ : C, 53.00: H, 4.45: N, 16.86. Found: C, 52.98: H, 4.43; N, 16.84

\section{4k. 6-Methyl-2-(pyrrolidin-1-yl)benzo[d]thiazole} m.p. $133{ }^{\circ} \mathrm{C}, \mathrm{m} / \mathrm{z}$ : 218.09; Mol. wt: 218.32; ${ }^{1} \mathrm{H}$ NMR $\left(400 \mathrm{MHz}, \mathrm{DMSO}-d_{6}\right): \delta=1.92\left(\mathrm{~d}, 4 \mathrm{H}\right.$, aliphatic $\left.-\mathrm{CH}_{2}-\right), 2.34$ (s, 3H, $\left.-\mathrm{CH}_{3}\right), 3.44$ (d, $4 \mathrm{H}, \mathrm{N}-\mathrm{CH}_{2}-$ ) , 8.01-8.62 (d, 3H, ArH), ${ }^{13} \mathrm{C}$ NMR $\left(75 \mathrm{MHz}, \mathrm{DMSO}-d_{6}\right): \delta=20.9,25.5,54.1,117.1$, 121.3, 126.6, 130.7, 134.1, 150.2, 168.0; Anal. calcd for $\mathrm{C}_{12} \mathrm{H}_{14} \mathrm{~N}_{2} \mathrm{~S}: \mathrm{C}, 66.02: \mathrm{H}, 6.46: \mathrm{N}, 12.83$. Found: $\mathrm{C}, 66.00: \mathrm{H}$, $6.44 ; \mathrm{N}, 12.81$.

\section{4l. 6-Methoxy-2-(pyrrolidin-1-yl)benzo[d]thiazole}

m.p. $130{ }^{\circ} \mathrm{C}, \mathrm{m} / \mathrm{z}$ : 234.08; Mol. wt: 234.32; ${ }^{1} \mathrm{H}$ NMR $\left(400 \mathrm{MHz}, \mathrm{DMSO}-d_{6}\right): \delta=1.92\left(\mathrm{~d}, 4 \mathrm{H}\right.$, aliphatic $\left.-\mathrm{CH}_{2}-\right), 3.44$ (d, $4 \mathrm{H}, \mathrm{N}-\mathrm{CH}_{2}-$ ), $3.83\left(\mathrm{~s}, 3 \mathrm{H},-\mathrm{OCH}_{3}\right), 7.00-7.53(\mathrm{~d}, 3 \mathrm{H}, \mathrm{ArH})$, ${ }^{13} \mathrm{C}$ NMR $\left(75 \mathrm{MHz}\right.$, DMSO- $\left.d_{6}\right): \delta=25.5,54.1,55.8,104.9$, 114.6, 118.2, 131.9, 145.5, 156.7, 168.0; Anal. calcd for $\mathrm{C}_{12} \mathrm{H}_{14} \mathrm{~N}_{2} \mathrm{OS}: \mathrm{C}, 61.51: \mathrm{H}, 6.02: \mathrm{N}, 11.96$. Found: $\mathrm{C}, 61.49: \mathrm{H}$, 6.00; N, 11.94 .
Table 1. Screening and control experiments. ${ }^{(a)}$

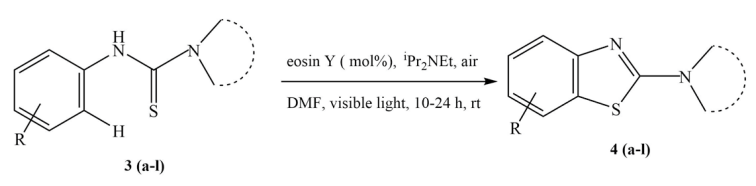

\begin{tabular}{cccccc}
\hline Entry & Visible Light & Eosin Y & Air & Time $/ \mathrm{h}$ & Yield $/ \%{ }^{(\mathrm{b})}$ \\
\hline 1 & + & + & + & 24 & $12^{(\mathrm{c})}$ \\
2 & + & + & + & 10 & 97 \\
3 & - & + & + & 24 & n.r..$^{(\mathrm{d})}$ \\
4 & + & - & + & 24 & n.r. \\
5 & + & + & - & 24 & n.r. \\
6 & + & + & + & 10 & $45^{(\mathrm{e})}$ \\
7 & + & + & $\mathrm{N}_{2}$ & 24 & trace \\
8 & + & + & $\mathrm{O}_{2}$ & 10 & 97 \\
9 & + & + & + & 10 & $97^{(\mathrm{f})}$ \\
10 & + & + & + & 10 & $60^{(\mathrm{g})}$ \\
11 & + & + & + & 10 & $42^{(\mathrm{h})}$ \\
\hline
\end{tabular}

(a) Reaction conditions: arylthiourea $(1.0 \mathrm{mmol})$, eosin $\mathrm{Y}(2.0 \mathrm{~mol} \%), \mathrm{iPr}_{2} \mathrm{NEt}$ ( 2.0 equiv.), DMF ( $3.0 \mathrm{~mL})$, green LEDs $2.4 \mathrm{~W}, 120 \mathrm{~lm}$ irradiation under an air atmosphere at rt.

(b) Isolated yield of the product (4a-I). n.r. = no reaction.

(c) The reaction was conducted without $\mathrm{Pr}_{2} \mathrm{NEt}$ base in DMF.

(d) The reaction was carried out in the dark.

(e) The reaction was carried out using $20 \mathrm{~W}$ CFL (compact fluorescent lamp).

(f) The reaction was carried out with 3.0 equiv. of $\mathrm{Pr}_{2} \mathrm{Net}$.

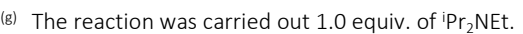

(h) The reaction was carried out with $1.0 \mathrm{~mol} \%$ of eosin $\mathrm{Y}$.

Table 2. Optimization of reaction conditions. ${ }^{(a)}$

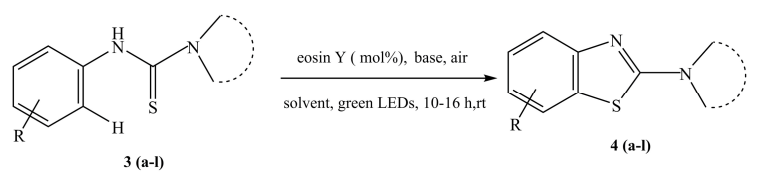

\begin{tabular}{cccccc}
\hline Entry & Eosin $\mathrm{Y} / \mathrm{mol} \%$ & Base & Solvent & Time $/ \mathrm{h}$ & Yield $/ \%$ (b) \\
\hline 1 & 3 & $\operatorname{Pr}_{2} \mathrm{NEt}$ & $\mathrm{DMF}$ & 10 & 97 \\
2 & 2 & $\mathrm{Pr}_{2} \mathrm{NEt}$ & $\mathrm{DMF}$ & 10 & 97 \\
3 & 1 & $\mathrm{Pr}_{2} \mathrm{NEt}$ & $\mathrm{DMF}$ & 10 & 42 \\
4 & 2 & $\mathrm{Pr}_{2} \mathrm{NEt}$ & $\mathrm{MeOH}$ & 16 & 72 \\
5 & 2 & $\mathrm{Pr}_{2} \mathrm{NEt}$ & $\mathrm{EtOH}$ & 16 & 62 \\
6 & 2 & $\mathrm{DBU}^{2}$ & $\mathrm{DMF}$ & 16 & 52 \\
7 & 2 & $\mathrm{DABCO}^{2}$ & $\mathrm{DMF}$ & 16 & 55 \\
8 & 2 & $\mathrm{Pr}_{2} \mathrm{NEt}$ & $\mathrm{DMSO}$ & 10 & 82 \\
9 & 2 & $\mathrm{Et}_{3} \mathrm{~N}$ & $\mathrm{DMF}$ & 16 & 65 \\
\hline
\end{tabular}

(a) Reaction conditions: arylthiourea $(1.0 \mathrm{mmol})$, eosin $\mathrm{Y}(2.0 \mathrm{~mol} \%)$, Base (2.0 equiv.), DMF ( $3.0 \mathrm{~mL}$ ), green LEDs $2.4 \mathrm{~W}, 120 \mathrm{~lm}$ irradiation under an air atmosphere at rt.

(b) Isolated yield of the product (4a-I). 


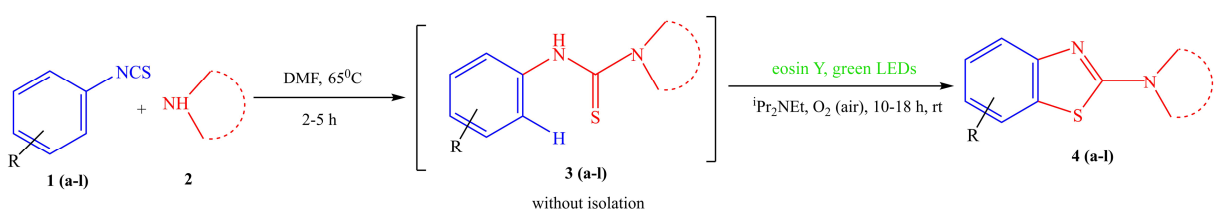

Scheme 2. One -pot facile synthesis of 2-aminobenzothiazole directly from arylisothiocyanate and secondary amine.

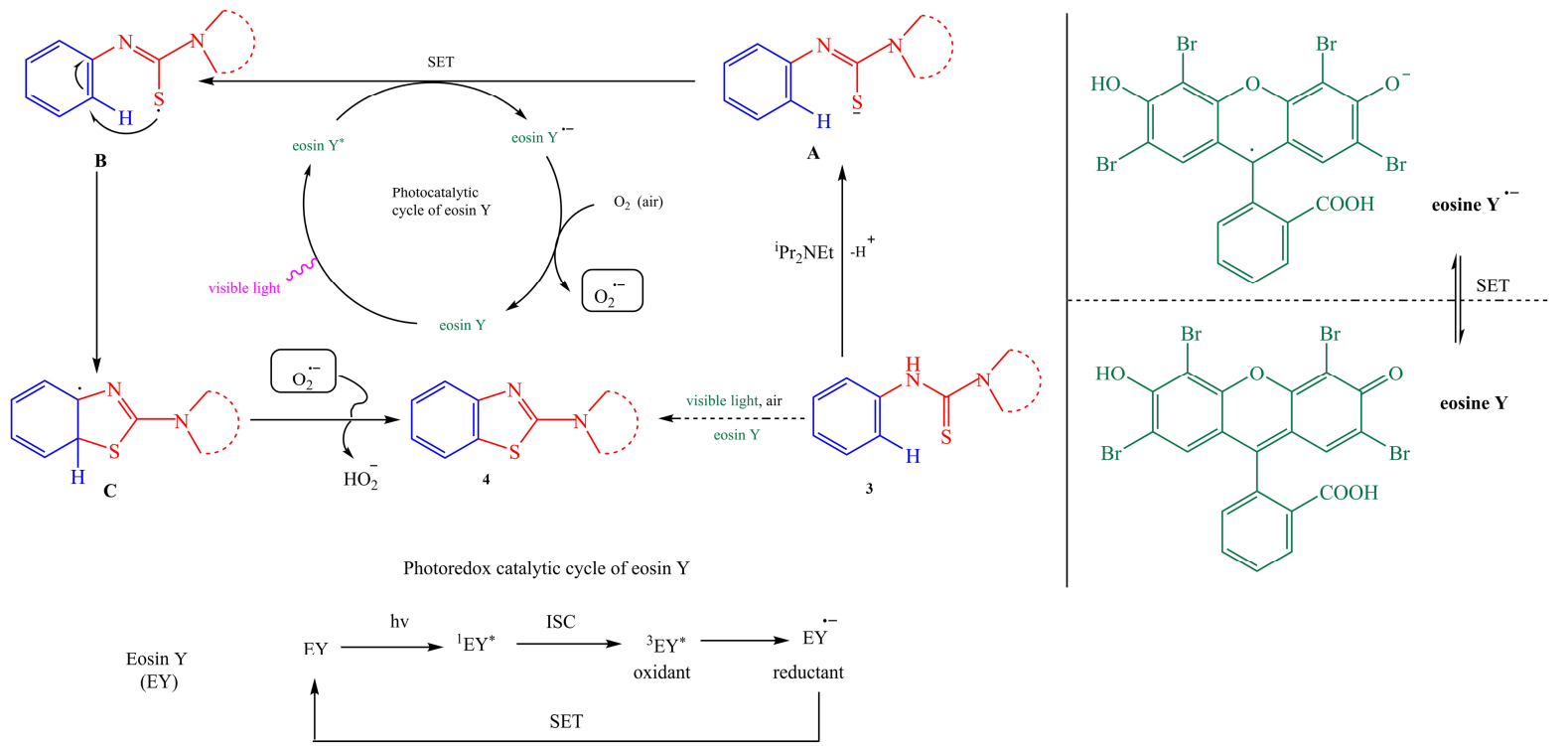

Scheme 3. Proposed mechanism for the visible light irradiated synthesis of 2-aminobenzothiazole using eosin $Y$ as photocatalyst.

\section{RESULTS AND DISCUSSION}

In order to work out the envisaged protocol, a key reaction was conducted with arylthiourea $\mathbf{3}(\mathrm{a}-\mathrm{I})$ in DMF containing $2 \mathrm{~mol} \%$ of eosin $\mathrm{Y}$ under an air atmosphere (without air bubbling) by irradiation with visible light (green light-emitting diodes (LEDs), $\lambda_{\max }=535 \mathrm{~nm}$ ) at rt. The reaction delivered the desired 2-aminobenzothiazole $\mathbf{4}(\mathrm{a}-\mathrm{I})$ in $12 \%$ isolated yield after $24 \mathrm{~h}$ (Table 1, entry 1 ). Following this experiment, a series of control experiments were performed, which indicates that an organic base is essential to give the desired product with high yield (97\%) (Table 1, entry 2 ) and $\mathrm{Pr}_{2} \mathrm{NEt}$ was found to be the best base (Table 2, entry 2 versus $6,7,9$ ). There was no product formation or it was formed in traces in the absence (-) of any one of the reagents / catalyst (Table 1 , entries 3-5). The reaction did not proceed satisfactorily when a household $20 \mathrm{~W}$ fluorescent lamp was used instead of green LEDs (Table 1, entries 6 versus 2). Notably, the same result was obtained on using $\mathrm{O}_{2}$ (balloon) instead of an air atmosphere (Table 1 , entry 8 versus 2 ), where as in the absence of any gas or under a nitrogen atmosphere no product formation was detected (Table 1, entry 5, 7). These results establish that visible light, base, photocatalyst and air all are essential (+) for the reaction and support the photocatalytic model of the reaction.

Next, the reaction conditions were optimized with respect to solvents and the catalyst used in the reaction. In all the tested solvents (DMF, DMSO, $\mathrm{MeOH}$ and $\mathrm{EtOH}$ ) the yield of $4(a-I)$ was $>55 \%$ (Table 2 ), which indicates that the reaction is not very sensitive to reaction media. DMF was the best solvent in terms of the reaction time and yield (Table 2 , entry 1 ), hence it was used throughout the synthesis. When the amount of the catalyst was decreased from $2 \mathrm{~mol} \%$ to $1 \mathrm{~mol} \%$, the yield of $4(\mathrm{a}-\mathrm{I})$ considerably reduced (Table 2, entry 3), but the use of $3 \mathrm{~mol} \%$ of the catalyst did not affect the yield (Table 2 , entry 1 ).

Under the established reaction conditions in hand, the reaction was tried in a one-pot procedure starting directly from an arylisothiocyanate $\mathbf{1}(\mathrm{a}-\mathrm{I})$ and a secondary amine (2) to give the desired product $\mathbf{4}(\mathrm{a}-\mathrm{I})$ as depicted in Scheme 2.

To our delight, it worked well and a number of symmetrical and unsymmetrical 2-aminobenzothiazoles were successfully synthesized starting directly from various arylisothiocyanate $\mathbf{1}(\mathbf{a}-1)$ and secondary amines (2) (Table 3). 
Table 3. Eosin $Y$ catalysed synthesis of 2-aminobenzothiazoles.

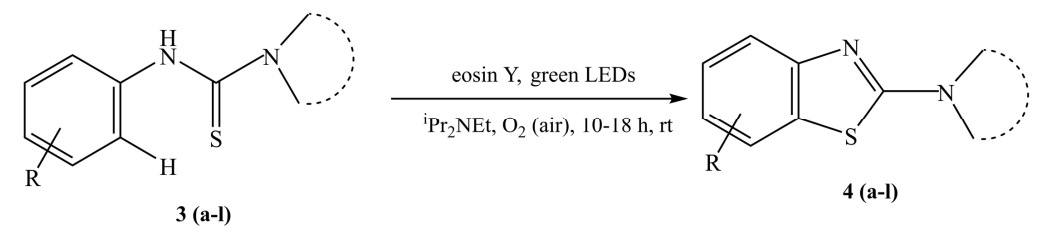

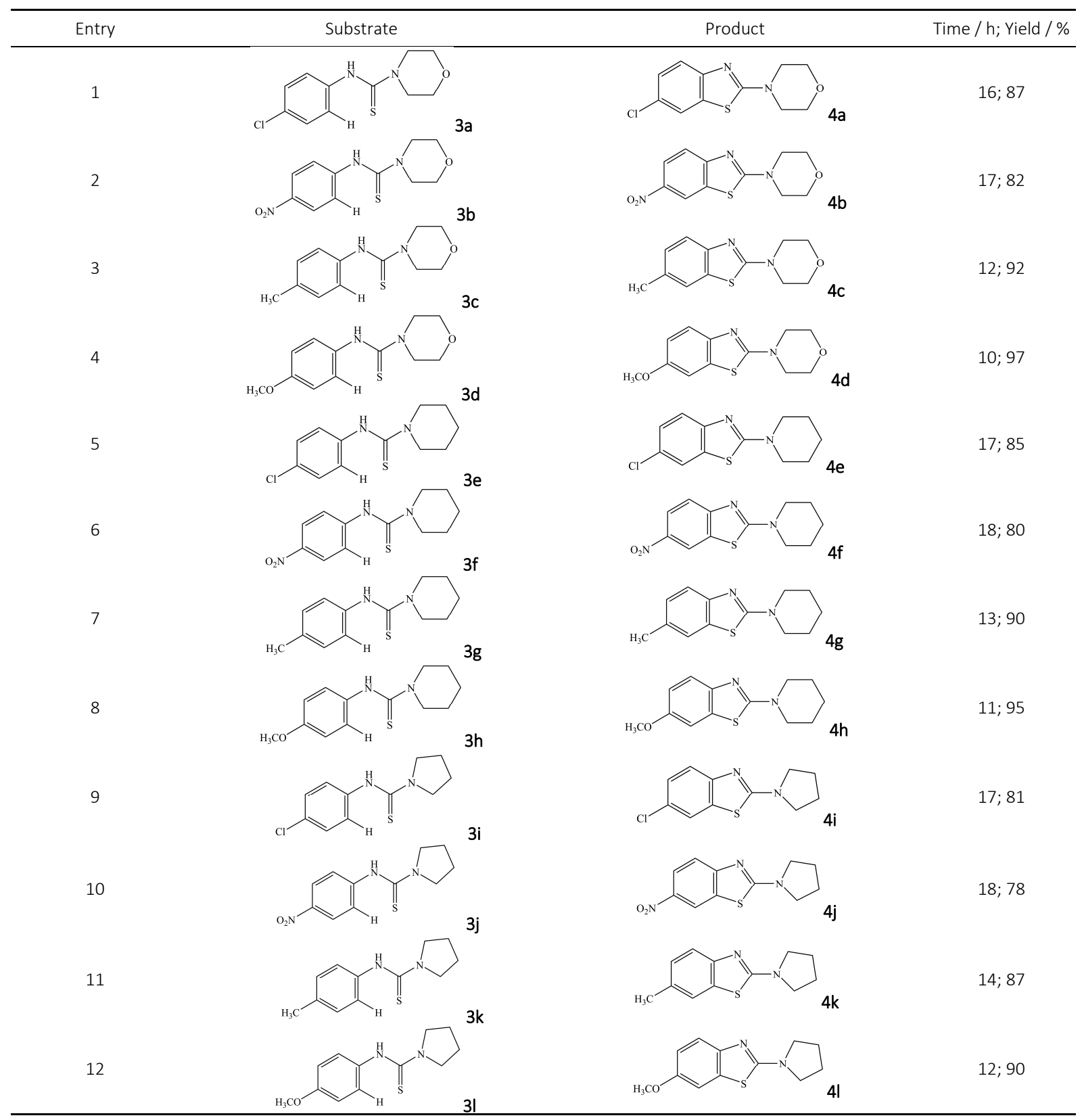

This clearly shows that the reaction is very mild and applicable to aryl and alkyl, tolerates considerable functional group variations like, $\mathrm{MeO}, \mathrm{Me}, \mathrm{Cl}$ and $\mathrm{NO}_{2}$ in the substrate $\mathbf{1}(\mathrm{a}-\mathrm{l})$, which results the desired product 4(a-l) in good to excellent yields (78-97\%). However, arylisothiocyanate (1) and a secondary amine (2) with an electron-donating group on the aromatic ring appear to react faster and afford marginally higher yields in comparison to those bearing an electron withdrawing group. 

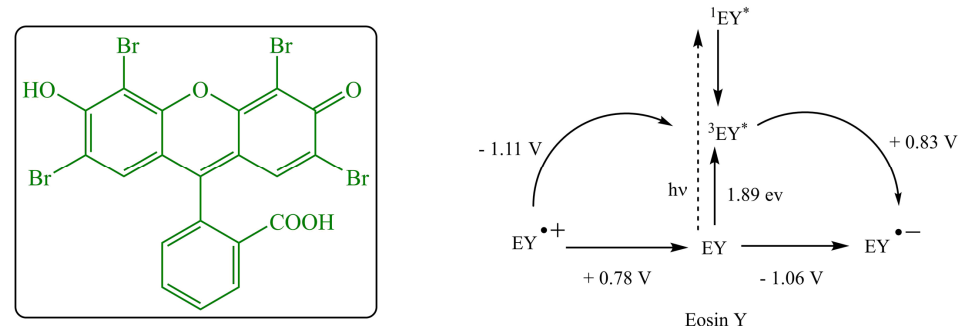

Scheme 4. The redox potentials of eosin $\mathrm{Y}$ in DMF- $\mathrm{H}_{2} \mathrm{O}(1: 1)$ in ground and corresponding excited states.

On the basis of the above observations and the literature precedents, a plausible mechanism involving photoredox catalysis for the oxidative cyclization of $\mathrm{N}$-arylthioureas is depicted in Scheme 3. On absorption of visible light, the organophotoredox catalyst eosin $\mathrm{Y}(\mathrm{EY})$ is excited to its singlet state ${ }^{1} \mathrm{EY}^{*}$ which through inter system crossing (ISC) comes to its more stable triplet state ${ }^{3} \mathrm{EY}^{*}$ and undergoes a single electron transfer (SET). ${ }^{3} \mathrm{EY}^{*}$ may undergo both reductive and oxidative quenching. ${ }^{[40-44]} \mathrm{A}$ SET from $A$ to ${ }^{3} \mathrm{EY}^{*}$ generates thioacyl radical $\mathrm{B}$, which undergoes intramolecular cyclization (5-endo-trig) to form $\mathrm{C}$ followed by attack of $\left(\mathrm{O}_{2}^{--}\right)$to give the product 4 , successively. The formation of superoxide radical anion $\left(\mathrm{O}_{2}^{-}\right)$during the reaction was confirmed by the detection of the resulting $\mathrm{H}_{2} \mathrm{O}_{2}$ using KI / starch indicator. ${ }^{[45]}$ The redox potential of eosin $\mathrm{Y}$ has also been incorporated in Scheme 4 for catalytic activity.

\section{CONCLUSION}

In conclusion, we have developed a novel organocatalysed method for the synthesis of 2-aminobenzothiazole directly from arylisothiocyanate and secondary amine in a one-pot procedure by using inexpensive eosin $Y$ as a powerful organophotoredox catalyst at $r$. The reaction involves visible light, a base and $\mathrm{O}_{2}$ (air) as a valuable reagents. This synthetic pathway includes a superior visible light promoted and Eosin $\mathrm{Y}$ organophotoredox catalysed methodology, which is superior in comparison to all other alternative synthetic methods for 2-aminobenzothiazole. This synthesis widens the scope of substrates for visible light photoredox reactions. The present methodology also offers many advantages of green chemistry such as high atom economy, reduced reaction time, one-pot consolidated procedure and high efficiency.

Acknowledgment. We sincerely thank SAIF, CDRI, Lucknow and IISC Banglaore for providing microanalyses and spectra.

\section{REFERENCES}

[1] J. W. Tucker. C. R. J. Stephenson, J. Org. Chem. 2012, $77,1617$.
[2] X. Sala, I. Romero, M. Rodriguez, L. Escriche, A. Llobet, Angew. Chem. Int. Ed. 2009, 48, 2842.

[3] O. Morton, Nature 2006, 443, 19.

[4] D. G. Nocera, Daedalus 2006, 135, 112.

[5] N. S. Lewis, Science 2007, 315, 798.

[6] D. Mandler, I. Willner, J. Am. Chem. Soc. 1984, 106, 5352.

[7] O. Ishitani, S. Yanagida, S. Takamuku, C. Pac, J. Org. Chem. 1987, 52, 2790.

[8] A. Inagaki, M. Akita, Coord. Chem. Rev. 2010, 254, 1220.

[9] C. K. Prier, D. A. Rankic, D. W. C. MacMillan, Chem. Rev. 2013, 113, 5322.

[10] D. A. Nicewicz, T. M. Nguyen, ACS Catal. 2014, 4, 355.

[11] J. Xie, H. Jina, P. Xu, C. Zhu, Tetrahedron Lett. 2014, $55,36$.

[12] X. Lang, X. Chen, J. Zhao, Chem. Soc. Rev. 2014, 43, 473.

[13] J. Hu, J. Wang, T. H. Nguyen, N. Zheng, Beilstein J. Org. Chem. 2013, 9, 1977.

[14] D. Rovelli, M. Fagnoni, A. Albini, Chem. Soc. Rev. 2013, 42, 97.

[15] T. P. Yoon, M. A. Ischay, J. Du, Nat. Chem. 2010, 2, 527.

[16] D. A. Nicewicz, D. W. C. MacMillan, Science 2008, 322, 77.

[17] M. A. Ischay, M. E. Anzovino, J. Du, T. P. Yoon, J. Am. Chem. Soc. 2008, 130, 12886.

[18] J. M. R. Narayanam, C. R. J. Stephenson, Chem. Soc. Rev. 2011, 40, 102.

[19] J. M. R. Narayanam, J. W. Tucker, C. R. J. Stephenson, J. Am. Chem. Soc. 2009, 131, 8756.

[20] X.-J. Yang, B. Chen, L.-Q. Zheng, L.-Z. Wu, C.-H. Tung, Green Chem. 2014, 16, 1082.

[21] Y. C. Teo, Y. Pan, C. H. Tan, ChemCatChem. 2013, 5, 235.

[22] K. Fidaly, C. Ceballos, A. Falguières, M. S.-I. Veitia, A. Guy, C. Ferroud, Green. Chem. 2012, 14, 1293.

[23] D.-T. Yang, Q.-Y. Meng, J.-J. Zhong, M. Xiang, Q. Liu, L.-Z. Wu, Eur. J. Org. Chem. 2013, 7528.

[24] Y.-Q. Zou, J.-R. Chen, X.-P. Liu, L.-Q. Lu, R. L. Davis, K. A. Jørgense, W.-J. Xiao, Angew. Chem. Int. Ed. 2012, $51,784$. 
[25] D. P. Hari, B. König, Org. Lett. 2011, 13, 3852.

[26] M. Neumann, S. Füldner, B. König, K. Zeitler, Angew. Chem. Int. Ed. 2011, 50, 951.

[27] V. Rey, S. M. Soria-Catro, J. E. Arguello, A. B. Peñéñory, Tetrahedron Lett. 50 (2009) 4720.

[28] G. Alang, R. Kaur, G. Kaur, A. Singh, P. Singla, Acta Pharm. Sci. 2010, 52, 213.

[29] E. Roduner, W. Kaim, B. Sarkar, V. B. Urlacher, J. Pleiss, R. Glaser, W.-D. Einicke, G. A. Sprenger, U. Beifuß, E. Klemm, C. Liebner, H. Hieronymus, S.-F. Hsu, B. Plietker, S. Laschat, ChemCatchem. 2013, 5, 82.

[30] Z. Shi, C. Zhang, C. Tang, N. Jiao, Chem. Soc. Rev. 2012, 41, 3381.

[31] D. P. Hari, T. Hering, B. König, Org. Lett. 2012, 14, 5334.

[32] S. Samanta, S. Das, P. Biswas, J. Org. Chem. 2013, 78, 11184.

[33] G. Crank, A. Mursydi, J. PhotoChem. Photobio. 1990, 53, 301.

[34] S. Gazi, R. Ananthakrishnan, RSC Adv. 2012, 2, 7781.

[35] J. Zhang, L. Wang, Q. Liu, Z. Yang, Y. Huang, Chem. Commun. 2013, 49, 11662.
[36] I. R. Siddiqui, P. K. Singh, V. Srivastava, J. Singh, Indian J. Chem. 2007, 46B, 1716.

[37] V. Srivastava, P. K. Singh, P. P. Singh, Chem. Heterocycl. Compd. 2014, 50, 573.

[38] V. Srivastava, P. K. Singh, P. P. Singh, Croat. Chem. Acta 2014, 87, 91.

[39] P. K. Singh, I. R. Siddiqui, Indian J. Chem. 2009, 48B, 1013.

[40] D. C. Neckers, O. M. Valdes- Aguilera, Adv. Photochem. 1993, 18, 315.

[41] M. V. Encinas, A. M. Rufs, S. G. Bertolotti, C. M. Previtali, Polymer 2009, 50, 2762.

[42] T. Lizarides, T. McCormick, P. Du, G. Luo, B. Lindley, R. Eisenberg, J. Am. Chem. Soc. 2009, 131, 9192.

[43] S. H. Lee, D. H. Nam, C. B. Park, Adv. Synth. Catal. 2009, 351, 2589.

[44] T. Xiao, X. Dong, Y. Tang, L. Zhou, Adv. Synth. Catal. 2012, 354, 3195.

[45] G. K. Fekarurhobo, S. S. Angaye, F. G. Obomann, J. Emerg. Trends Engg. Appl. Sci. 2013, 4, 394. 